\title{
Promising application of monoclonal antibody against chikungunya virus E1- antigen across genotypes in immunochromatographic rapid diagnostic tests
}

Keita Suzuki ${ }^{1,2}$, Ralph Huits ${ }^{3}$, Juthamas Phadungsombat ${ }^{4}$, Aekkachai Tuekprakhon ${ }^{4,5}$, Emi E. Nakayama ${ }^{1}$, Riemsdijk van den Berg ${ }^{6}$, Barbara Barbé ${ }^{3}$, Lieselotte Cnops ${ }^{3}$, Rummana Rahim7, Abu Hasan ${ }^{7}$, Hisahiko Iwamoto ${ }^{2}$, Pornsawan Leaungwutiwong ${ }^{5}$, Marjan van Esbroeck ${ }^{3}$, Mizanur Rahman ${ }^{7}$ and Tatsuo Shioda ${ }^{1,4^{*}}$

\begin{abstract}
Background: Three different genotypes of chikungunya virus (CHIKV) have been classified: East/Central/South African (ECSA), West African (WA), and Asian. Previously, a rapid immunochromatographic (IC) test detecting CHIKV E1-antigen showed high sensitivity for certain ECSA-genotype viruses, but this test showed poor performance against the Asian-genotype virus that is spreading in the American continents. We found that the reactivity of one monoclonal antibody (MAb) used in the IC rapid diagnostic test (RDT) is affected by a single amino acid substitution in E1. Therefore, we developed new MAbs that exhibited specific recognition of all three genotypes of CHIKV.

Methods: Using a combination of the newly generated MAbs, we developed a novel version of the IC RDT with improved sensitivity to Asian-genotype CHIKV. To evaluate the sensitivity, specificity, and cross-reactivity of the new version of the IC RDT, we first used CHIKV isolates and E1-pseudotyped lentiviral vectors. We then used clinical specimens obtained in Aruba in 2015 and in Bangladesh in 2017 for further evaluation of RDT sensitivity and specificity. Another alphavirus, sindbis virus (SINV), was used to test RDT cross-reactivity.
\end{abstract}

Results: The new version of the RDT detected Asian-genotype CHIKV at titers as low as 10^4 plaque-forming units per $\mathrm{mL}$, a concentration that was below the limit of detection of the old version. The new RDT had sensitivity to the ECSA genotype that was comparable with that of the old version, yielding 92\% (92 out of 100) sensitivity (95\% confidence interval 85.0-95.9) and 100\% (100 out of 100) specificity against a panel of 100 CHIKV-positive and 100 CHIKV-negative patient sera obtained in the 2017 outbreak in Bangladesh.

(Continued on next page)

\footnotetext{
* Correspondence: shioda@biken.osaka-u.ac.jp

${ }^{1}$ Research Institute for Microbial Diseases, Osaka University, Suita, Japan

${ }^{4}$ Mahidol-Osaka Center for Infectious Diseases, Mahidol University, Bangkok,

Thailand

Full list of author information is available at the end of the article
}

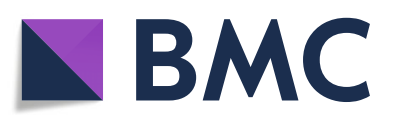

(- The Author(s). 2020 Open Access This article is licensed under a Creative Commons Attribution 4.0 International License, which permits use, sharing, adaptation, distribution and reproduction in any medium or format, as long as you give appropriate credit to the original author(s) and the source, provide a link to the Creative Commons licence, and indicate if changes were made. The images or other third party material in this article are included in the article's Creative Commons licence, unless indicated otherwise in a credit line to the material. If material is not included in the article's Creative Commons licence and your intended use is not permitted by statutory regulation or exceeds the permitted use, you will need to obtain permission directly from the copyright holder. To view a copy of this licence, visit http://creativecommons.org/licenses/by/4.0/. The Creative Commons Public Domain Dedication waiver (http://creativecommons.org/publicdomain/zero/1.0/) applies to the data made available in this article, unless otherwise stated in a credit line to the data. 
(Continued from previous page)

Conclusions: Our newly developed CHIKV antigen-detecting RDT demonstrated high levels of sensitivity and lacked cross-reactivity against SINV. These results suggested that our new version of the CHIKV E1-antigen RDT is promising for use in areas in which the Asian and ECSA genotypes of CHIKV circulate. Further validation with large numbers of CHIKV-positive and -negative clinical samples is warranted. (323 words).

Keywords: Chikungunya virus, E1 protein, Rapid immunochromatographic RDT, Monoclonal antibody, ECSA, Asian genotype

\section{Introduction}

Chikungunya virus (CHIKV) is a mosquito-transmitted alphavirus belonging to the family Togaviridae. This pathogen was first isolated in Tanzania, East Africa, in 1952 [1, 2]. Since then, CHIKV has caused sporadic outbreaks throughout the African and Asian continents. Although only one serotype CHIKV exists, the virus is classified into three genotypes named after the geographical location where the respective genotype was first recognized: East/Central/South African (ECSA), West African (WA), and Asian [3, 4]. CHIKV was considered a neglected tropical pathogen until a large outbreak was reported on Indian Ocean islands in 2005. The outbreak affected 244,000 individuals, one-third of the total population in this region $[5,6]$. Sequence analysis revealed a specific amino acid change that rendered the virus infective to Aedes albopictus; this clade of the ECSA genotype therefore was designated as the Indian Ocean Lineage (ECSA-IOL) [6, 7]. Subsequently, ECSAIOL also was identified in India [8] and South East Asia (including Thailand [9], Cambodia [10], and Malaysia [11]), and was detected for the first time in European countries (including Italy [12] and France [13]). In contrast, non-IOL ECSA-genotype CHIKV was detected in Brazil in 2013 [14, 15]. Asian-genotype CHIKV subsequently gave rise to a novel clade, designated as Asian/ American [16, 17]. This clade has become a public health problem in the Caribbean islands and the Central American mainland [18, 19]. Worldwide, approximately one billion people are estimated to live in areas at risk of CHIKV outbreaks [20].

More than $75 \%$ of CHIKV-infected individuals develop acute febrile symptoms such as high fever, headache, and muscle and joint pains $[21,22]$ that are similar to those observed in patients infected by other mosquitoborne viruses such as dengue dengue and Zika viruses [23]. However, the CHIKV illness often is associated with prolonged and incapacitating arthritis; for this reason, large CHIKV epidemics have considerable economic consequences, highlighting their significant public health impact. To distinguish CHIKV infection in the acute (viremic) phase, several nucleic acid detection methods are considered the gold standards [24]. However, immunochromatographic (IC) rapid diagnostic tests (RDTs) are user-friendly and easy to store without the need to maintain a cold chain, while facilitating early diagnosis. Such RDTs are expected to increase the accessibility of laboratory diagnosis of CHIKV infection. Previously, Okabayashi et al. reported a prototype IC RDT with high sensitivity ( 68 out of $76,89.4 \%$ ) and specificity (34 out of 36, 94.4\%) against clinical samples known to contain ECSA-genotype CHIKV in Thailand and Laos [25]. That RDT also showed high sensitivity (74 out of $79,93.7 \%$ ) and specificity (42 out of $44,95.5 \%$ ) in India [26]. However, another trial of that RDT with confirmed clinical samples of Asian-genotype CHIKV from the island of Aruba revealed low sensitivity (10 out of 30, 33.3\%) [27].

We previously showed that CK47, one of the monoclonal antibodies (MAbs) used in the RDT described by Okabayashi et al., has a limited ability to recognize the Asian-genotype E1 protein [28]. Notably, the Asiangenotype CHIKV encodes an aspartic acid (D) at residue 284 of the predicted E1 protein (residue 350 of the $6 \mathrm{~K}$ E1 polyprotein), a position that is encoded as a glutamic acid (E) in the predicted E1 proteins of certain ECSAgenotype CHIKVs [28]. This polymorphism critically affected the utility of that diagnostic tool across the various CHIKV genotypes [17]. To improve the sensitivity and specificity of the IC RDT, we obtained several antiCHIKV MAbs targeting the E1 and capsid (CA) proteins of CHIKV [29]. These MAbs were shown to exhibit broad reactivity towards all genotypes of CHIKV, and some of these MAbs lacked cross-reactivity to other alphaviruses, including eastern, western, and Venezuela equine encephalitis viruses. Here, we report the development and evaluation of an improved CHIKV antigendetection diagnostic test that incorporates these new MAbs.

\section{Materials and methods}

\section{Screening of MAbs against CHIKV E1 and CA}

To select anti-E1 MAbs suitable for use in an IC RDT, we screened 7 anti-E1 MAbs (3D11, 9E3, 11E11, 13H11, 15B2, 19B8, and RC-5) generated previously [29] by adsorbing each anti-E1 MAbs onto nitrocellulose membranes. Six of these MAbs (excepting 15B2) were conjugated with gold nanoparticles (AuNPs); 15B2 was excluded because that 
MAb is isotype IgG2b [29], which is difficult to conjugate with AuNPs (unpublished observations). All combinations of anti-E1 MAbs were tested by placing dipsticks in microcentrifuge tubes or the wells of a 96-well plate containing (per tube or well) $60 \mu \mathrm{L}$ of extraction buffer and $30 \mu \mathrm{L}$ of formaldehyde-inactivated culture supernatant of CHIKV S27-infected Vero cells. After $15 \mathrm{~min}$, a C10066-10 IC Reader (Hamamatsu Photonics K.K., Japan) was used to quantitate the intensities of the signals, and results were expressed as signal/background (the intensities of the signals without CHIKV antigens) ratios. This procedure was repeated another three times using recombinant CHIKV E1 protein (Aalto Bio Reagents Ltd., Dublin, Ireland), culture supernatant from Vero cells infected with Sendai virus expressing Asian CHIKV CK12 6 K-E1protein [29], and culture supernatant of CHIKV CP10-infected Vero cells. In the case of anti-CA MAbs, all five MAbs (24B3, 26A2, 32A3, 37C7, and 41G5) [29] were able to be conjugated with AuNP and evaluated by the procedure described for anti-E1 MAbs using culture supernatant of CHIKV CP10infected Vero cells as the antigen.

\section{Assembly and evaluation of IC RDT}

The details of components of the 1st version (version A) have been described previously [25]. Briefly, the rapid IC RDT incorporated two mouse anti-CHIKV MAbs: CK47 was immobilized onto the membrane at the test line and used for CHIKV antigen-capture; CK119 was conjugated to AuNPs and placed at the conjugated pad by TANAKA Kikinzoku Kogyo K. K, Japan. The 2nd (versions $\mathrm{B}, \mathrm{C}, \mathrm{D}, \mathrm{E}, \mathrm{F}, \mathrm{M}, \mathrm{N}$ ) and 3rd (version $\mathrm{O}$ ) -generation IC RDTs were assembled with the combinations of MAbs shown in Table 1.

Thirty microliters of serially diluted culture supernatant containing CHIKV or CHIKV-pseudotyped lentiviral vector at various concentrations were mixed with $60 \mu \mathrm{L}$ IC RDT extraction buffer in a tube. The IC dipstick then was inserted into the tube of diluted supernatant to start the reaction. After $15 \mathrm{~min}$, the appearance of the control and test lines was assessed.
An IC Reader also was used to quantitate the intensities of the test lines; values were expressed as milliabsorbance units (mAbs).

\section{Cells and viruses}

African green monkey kidney epithelium (Vero) cells (ATCC CCL-81) and baby hamster kidney (BHK) cells (ATCC CCL-10) were maintained in Minimum Essential Medium (MEM; Life Technologies, Inc.) supplemented with $10 \%(\mathrm{v} / \mathrm{v})$ heat-inactivated fetal bovine serum (FBS; Life Technologies, Inc.). Human Embryonic Kidney (HEK293T) cells were maintained in Dulbecco's Modified Eagle Medium (DMEM; Life Technologies, Inc.) supplemented with $10 \%(\mathrm{v} / \mathrm{v})$ heat-inactivated FBS. These cells were maintained at $37^{\circ} \mathrm{C}$ in a $5 \% \mathrm{CO}_{2}$ environment. For culturing cells that had been infected with CHIKV, the concentration of FBS in the medium was reduced to $2 \%$. Aedes albopictus-derived C6/36 cells were maintained at $28^{\circ} \mathrm{C}$ in L-15 medium (Life Technologies, Inc.) supplemented with $2 \%$ heat-inactivated FBS and $0.3 \%$ tryptose phosphate broth.

The ECSA-genotype CHIKV strain CP10 was isolated during a 2010 outbreak in Thailand [30, 31]; Asiangenotype strains ARUBA-15801125 (NCBI Accession Number LC500216), ARUBA-15800567 (LC500220), and ARUBA-15801654 (LC500221) were isolated from the sera of patients in Aruba [27, 32, 33]. Sindbis virus (SINV; strain R68), another alphavirus, was propagated in BHK cells. CHIKV strains were propagated using Vero and C6/36 cells. For viral titration, a standard plaque assay was performed [34].

\section{CHIKV-pseudotyped lentiviral vector}

The production of the CHIKV-pseudotyped lentiviral vectors was performed as described previously [35], with some modifications. Briefly, the process employed three essential plasmids: a pCAGGS MSII derivative that harbors a DNA cassette encoding the Asian-genotype CHIKV E3-E2-6K-E1 polyprotein corresponding to the CK12-686 strain [28]; pLenti CMV Puro LUC (w168-

Table 1 List of antibody combinations

\begin{tabular}{llll}
\hline Version & Detection antibody (gold labeled) & Capture antibody (membrane bound) & Target \\
\hline A & CK119 & CK47 & E1 \\
B & $13 \mathrm{H} 11$ & $3 \mathrm{D} 11+15 \mathrm{~B} 2$ & E1 \\
C & $41 \mathrm{G} 5$ & $26 \mathrm{~A} 2$ & Capsid \\
D & $41 \mathrm{G} 5$ & $24 \mathrm{~B} 3$ & Capsid \\
E & $13 \mathrm{H} 11+11 \mathrm{~F} 11$ & $15 \mathrm{~B} 2$ & E1 \\
F & $26 \mathrm{~A} 2+41 \mathrm{G} 5$ & $24 \mathrm{~B} 3$ & Capsid \\
M & $26 \mathrm{~A} 2+41 \mathrm{G} 5$ & $32 \mathrm{~A} 3$ & Capsid \\
N & $13 \mathrm{H} 11+26 \mathrm{~A} 2+41 \mathrm{G} 5$ & $15 \mathrm{~B} 2+32 \mathrm{~A} 3$ & E1 + capsid \\
O & $13 \mathrm{H} 11+$ CK1 19 & $3 \mathrm{D} 11+15 \mathrm{~B} 2+$ CK47 & E1 \\
\hline
\end{tabular}


1), which carries a reporter gene encoding firefly luciferase (Addgene, Cambridge, MA); and psPAX2, a lentivirus packaging vector. All three plasmids were transfected into HEK293T cells using polyethelenimine (Polysciences, Inc., Warrington, PA) [35]. Culture medium was replaced with fresh medium after $6 \mathrm{~h}$; the spent medium then was collected at $48 \mathrm{~h}$ after transfection. The titer of lentivector was determined using the RETROtek HIV-1 p24 Antigen-ELISA kit (Zeptometrix; Buffalo, NY), according to manufacturer's instructions. For production of WA- and ECSA-genotype E1s, we used pCAGGS MSII derivatives encoding (respectively) the E3-E2-6K-E1 sequence of WA-genotype strain 37, 997 or a chimeric E3-E2-6K-E1 sequence composed of sequences from strain 379,971 E3-E2 and strain CP10 6 K-E1.

\section{Clinical samples}

The Asian-genotype CHIKV serum panel was obtained for evaluation of the 2nd-generation version-B RDT from CHIKV-specific RT-PCR-positive $(n=26)$ and -negative $(n=54)$ samples of patients who had presented with febrile illness during the 2014-15 CHIKV epidemic in Aruba. These sera had been collected at 3-10 days (median 7 days) after the onset of symptoms. Median $\mathrm{Ct}$ value (and its interquartile range, IQR) of RT-PCRpositive samples were 35.29 (34.22-35.50). Forty samples were shown to be anti-CHIKV IgM- or IgG-positive by testing in Aruba using the Anti-Chikungunya Virus IgM/IgG IIFT assay (Euroimmun, Lübeck, Germany). Among this sample subset, 34 were anti-CHIKV IgMpositive and 31 were IgG-positive ( 25 were both antiCHIKV IgM- and IgG-positive). Samples then were stored at $-80^{\circ} \mathrm{C}$ until shipped on dry ice to Institute of Tropical Medicine, Antwerp (ITM), where CHIKVspecific RT-PCR testing was performed [32]. After this testing, these specimens were stored at $-80^{\circ} \mathrm{C}$ until evaluation by the E1-antigen IC RDT (i.e., after being subjected to a total of 2 freeze-thaw cycles). The protocol was approved by the Institutional Review Board of the ITM (1013/15).

A second set of clinical samples was obtained from the Apollo Hospitals Dhaka, Bangladesh. In 2017, a sudden increase in febrile cases was observed in Dhaka City, Bangladesh. Between July 2017 and February 2018, 1688 patients visited Apollo Hospitals Dhaka, with acute onset of fever (within 7 days from onset), myalgia, arthralgia, and headache; some patients also experienced a maculopapular rash and/or gastrointestinal symptoms. Out of 1688 febrile cases, 643 (38.1\%) and 269 (15.9\%) were laboratory-confirmed as chikungunya fever and dengue fever, respectively, by RT-PCR with the simultaneous detection and differentiation of CHIKV and dengue virus (DENV) (Fast-Track Diagnostics, Luxembourg). Notably, the sequences of CHIKV in this season in Dhaka (NCBI Accession No. LC364266-LC364269) were reported as ECSA genotype [36], encoding a $6 \mathrm{~K}-\mathrm{E} 1$ polyprotein with an $\mathrm{E}$ at residue 350 (corresponding to residue 284 in the mature E1 protein). These CHIKVs lacked an alanineto-valine substitution at residue 226 of the E1. CHIKV PCR-positive or DENV PCR-positive sera were stored at $-80^{\circ} \mathrm{C}$. The titer of serum IgM against CHIKV was measured using an ELISA kit (MBS495200) purchased from MyBioSource (San Diego, CA, USA). One hundred CHIKV RT-PCR-positive and 100 DENV RT-PCRpositive sera were used for evaluation of the 3rdgeneration version-O RDT. The median $\mathrm{Ct}$ value (IQR) of CHIKV RT-PCR-positive samples was 17.02 (14.8919.92). The study proposal was approved by the research and ethics committee of Apollo Hospitals Dhaka (ERC $16 / 2018-1)$.

\section{Results}

\section{Selection of MAbs against CHIKV E1 and CA in the 2nd} generation of RDT

We began our study by performing screening of the 7 anti-E1 MAbs that we had generated previously [29]. We used four different antigens for this screen, as described in Materials and Methods. Mean and standard deviations of signal/background ratios are shown in panel A of Additional file 1. Thirty-three of the 36 tested combinations yielded low signal/background ratios in the context of immunochromatography. One combination showed high levels of variation and only two combinations showed signal/background ratios above 2.0. In the case of 5 anti-CA MAbs [29], more than half of the combinations (16 out of 20 ) gave signal/background ratios above 2.0 when tested against the culture supernatant of CHIKV CP10-infected Vero cells (panel B of Additional file 1). Therefore, we chose the combinations of anti-E1 MAbs (IC RDT versions B and E) and antiCA MAbs (IC RDT versions $\mathrm{C}, \mathrm{D}, \mathrm{F}$, and $\mathrm{M}$ ) to construct trial IC RDTs (Table 1).

\section{Sensitivity of the 2nd-generation IC RDTs to ECSA- and Asian-genotype CHIKV isolates and CHIKV envelope- pseudotyped viruses}

To evaluate the reactivity of these new CHIKV antigen detection RDTs, we used four dilutions of ECSA- and Asian-genotype viruses, at titers of $10^{\wedge} 7,10^{\wedge} 6,10^{\wedge} 5$, and $10^{\wedge} 4 \mathrm{PFU} / \mathrm{mL}$. The limit of visible detection (i.e., with naked eyes) of CHIKV protein was $15 \mathrm{~m}$-absorbance units (mAbs) of color intensity as measured by an IC Reader. All rapid IC strips gave valid results, as indicated by the presence of the control lines. Quantitative measurement of the intensities of the test lines revealed that the E1 antigen-detecting RDT version B showed the best performance in detection of Asian-genotype CHIKV 
(Fig. 1, Additional file 2). At higher concentrations of $\mathrm{CHIKV}$, the gold-conjugated antibody in versions $\mathrm{B}$ and E tended to aggregate, thereby impeding quantifiability (Fig. 1 and Additional file 2). In the case of ECSAgenotype $\mathrm{CHIKV}$, the previous version-A RDT exhibited the best performance (Fig. 1). In contrast to the E1 detection RDTs, CA detection RDTs displayed similar reactivity to both $\mathrm{CHIKV}$ genotypes. Nevertheless, none of the CA detection RDTs was as sensitive as the E1 detection RDTs, especially at $10^{\wedge} 5 \mathrm{pfu} / \mathrm{mL}$ of CHIKV. Furthermore, addition of anti-CA antibodies to an E1 detection RDT failed to improve the RDT sensitivity (RDT version $\mathrm{N}$ in Additional file 2). We therefore decided to use E1 as the target of the IC RDT. Comparison

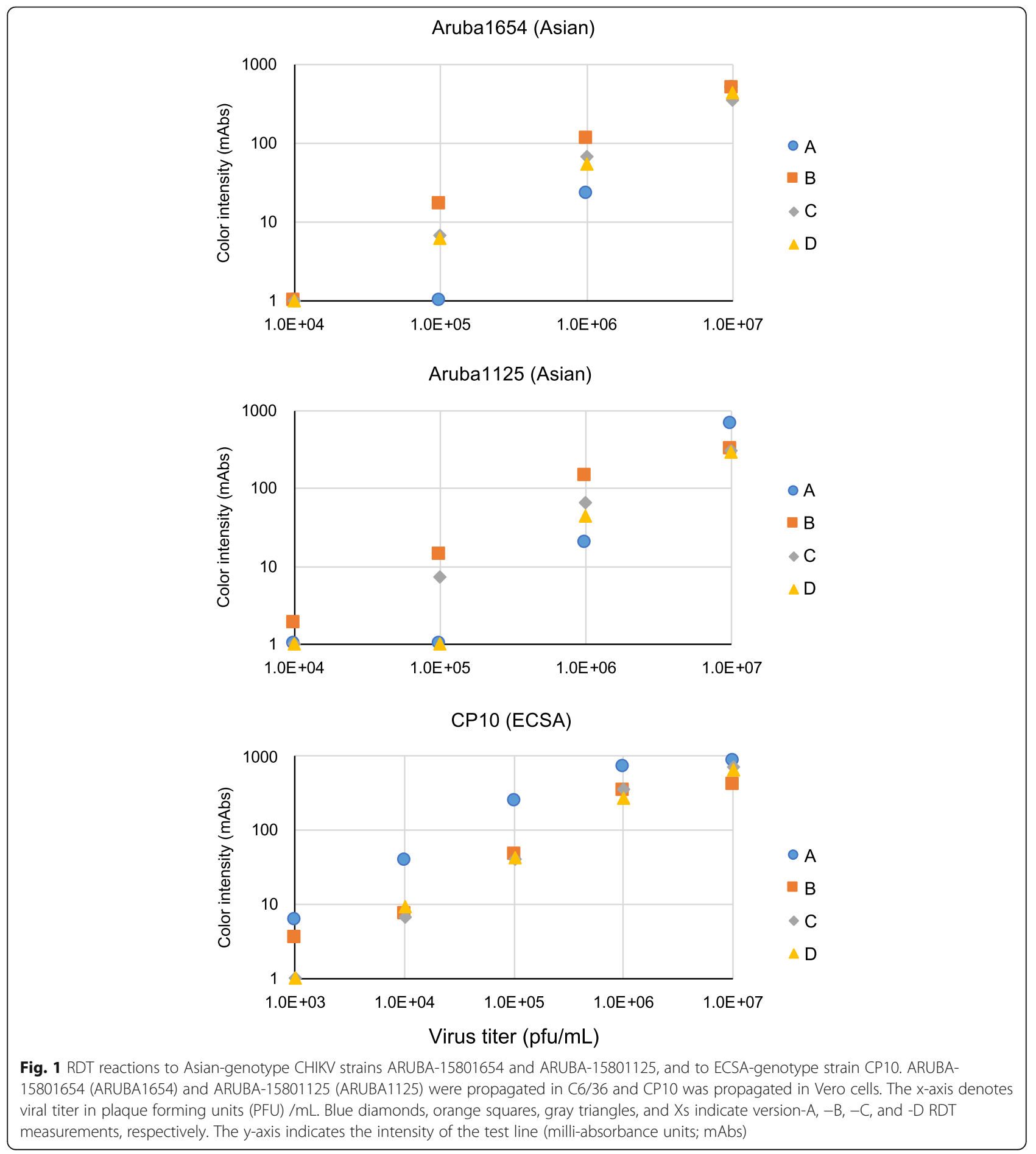


of sensitivities between versions $B$ and $E$ showed that gold-conjugated antibodies in version $\mathrm{E}$ yielded more aggregates at $10^{\wedge} 7 \mathrm{pfu} / \mathrm{mL}$ of $\mathrm{CHIKV}$, especially against Asian-genotype viruses. We therefore decided to stop further development of RDT version E.

We next examined the sensitivity of the version- $B$ RDT to WA-genotype CHIKV. Since we do not have access to live CHIKV of the WA genotype, we used CHIKV envelope-pseudotyped lentivirus to evaluate the IC RDT sensitivity. As shown in Fig. 2, the version-B RDT showed higher sensitivity than did the 1st version RDT (version A) for detection of WA-genotype virus along with Asian-genotype virus. However, as we anticipated from the results shown in Fig. 1, sensitivity of the version-B RDT was not comparable to that of the version-A RDT for detection of ECSA-genotype CHIKV.

Trial of 2nd-generation RDT version B with sera from Asian-genotype CHIKV patients

An Asian-genotype CHIKV outbreak occurred on the Caribbean island of Aruba in 2014-2015. We previously evaluated the version-A RDT using Aruban clinical samples of Asian-genotype CHIKV and found low sensitivity (33.3\%) for these specimens [27]. We performed an evaluation of the sensitivity of our the new generation of the RDT with 80 samples (40 anti-CHIKV IgM- and IgG-negative, 40 anti-CHIKV IgM- or IgG-positive). Individual data for the Aruba island patients are summarized in Additional file 3. Among the 80 samples, only 5 CHIKV RT-PCR-positive and IgG- and IgM-negative samples had been obtained. The 2nd-generation RDT version B detected 4 out of 5 of these samples (80, 95\% confidence interval (95\%-CI) 37.6-96.4), as shown in Table 2. The sensitivity was lower in the IgG-/IgM-positive group (7 of 21 (33.3, 95\%-CI 17.2-54.6)). Similarly, specificity was high (33 out of 35 (94.3, 95\%-CI 81.498.4)) in the IgG- and IgM-negative group and lower in the IgG-/IgM-positive group (12 of 19 (63.2, 95\%-CI 41.0-80.9)). The sensitivity and specificity did not change significantly in the 34 IgM-positive and 31 IgGpositive groups (Additional file 4).

Of those 80 samples, a subset of 20 also was tested using the previous RDT version A in order to permit side-by-side comparison with the version-B RDT. These 20 (10 PCR-positive and 10 PCR-negative) samples comprised 6 that were IgG- and IgMnegative and 14 that were IgG- or IgM-positive (Additional files 3 and 5). The version-A RDT failed to detect any of these samples, while the version-B RDT detected 4 out of 10 PCR-positive samples (40\%). Sensitivity and specificity of the version-B RDT for these 20 samples were comparable to those for the remaining 60 samples (Additional file 6).
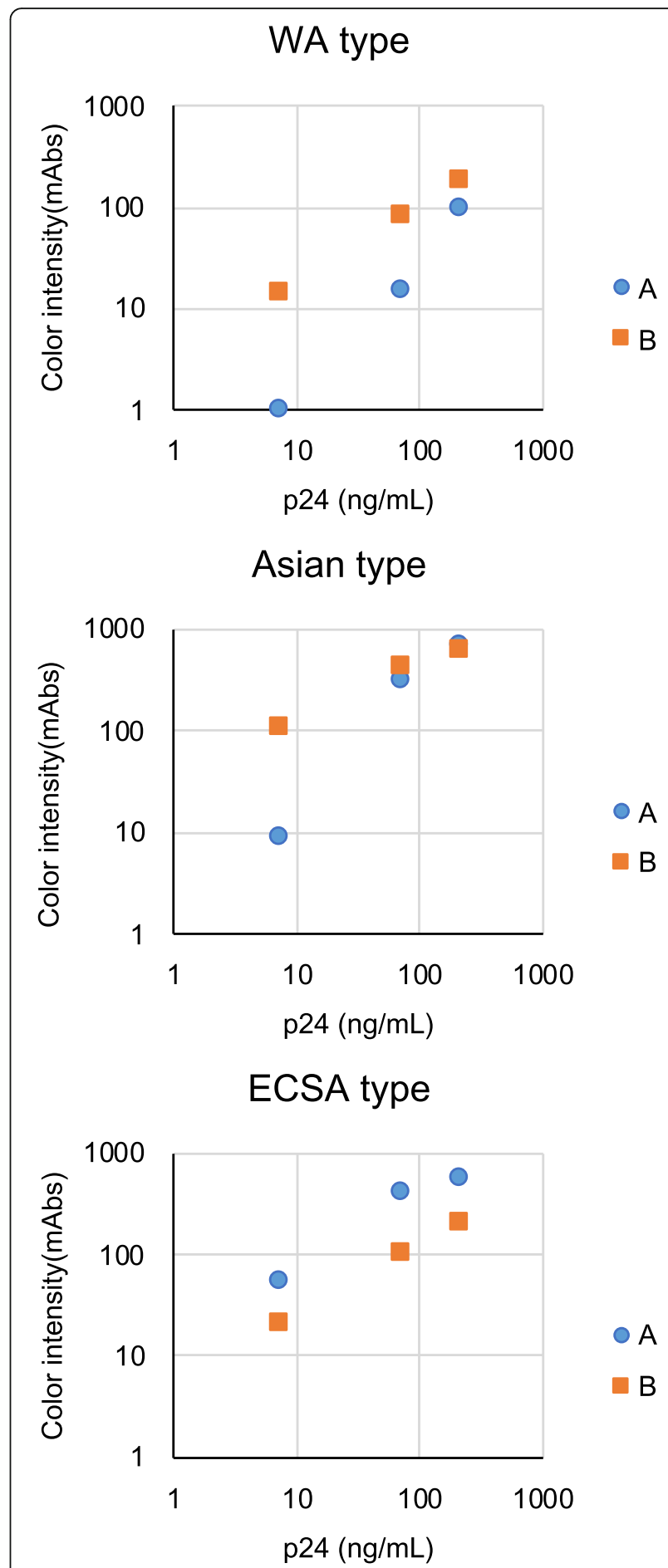

Fig. 2 RDT reaction to pseudotyped lentiviruses bearing CHIKV envelope proteins. Pseudotyped lentiviruses bearing CHIKV envelope proteins from ECSA-genotype strain CP10, Asian-genotype strain CK12-686 and WA-genotype strain 37,997 were prepared at three concentrations of HIV-1 capsid protein (210, 70, and $7 \mathrm{ng} / \mathrm{mL})$. Blue circles and orange squares indicate version- $A$ and $-B$ RDT measurements, respectively. The $y$-axis indicates the intensity of the test line (milli-absorbance units; mAbs) 
Table 2 Evaluation of 2nd-generation CHIKV E1 detection RDT version $B$ in clinical samples

\begin{tabular}{lllllll}
\hline IgG/ & PCR & \multicolumn{2}{l}{ IC RDT } & Sensitivity & Specificity & OAA \\
\cline { 3 - 5 } & & $(+)$ & $(-)$ & & & \\
\hline$(-)$ & $(+)$ & 4 & 1 & 80.0 & & 92.5 \\
& $(-)$ & 2 & 33 & & 94.3 & \\
$(+)$ & $(+)$ & 7 & 14 & 33.3 & & 47.5 \\
& $(-)$ & 7 & 12 & & 63.2 & \\
Total & $(+)$ & 11 & 15 & 42.6 & & 70.0 \\
& $(-)$ & 9 & 45 & & 83.3 & \\
\hline
\end{tabular}

$\operatorname{lgG} / \lg M(-)$ : anti-CHIKV IgM- and lgG-negative cases IgG/IgM (+): anti-CHIKV IgM- or IgG-positive cases

Sensitivity: percentage of matches between results of PCR and IC RDT in PCR positive samples

Specificity: percentage of matches between results of PCR and IC RDT in PCR negative samples

$O A A$ overall agreement (percentage of total matches between results of PCR and IC RDT)

Sensitivity of the 3rd-generation IC RDT version $\mathrm{O}$ for ECSA- and Asian-genotype CHIKV isolates and CHIKV envelope-pseudotyped viruses

Although the version-B RDT showed improved sensitivity for Asian-genotype CHIKV, the sensitivity of the version-B RDT to ECSA-genotype CHIKV was not comparable to that of the version-A RDT (Figs. 1 and 2). To address this issue, we combined the antibodies used in the version-A and - B RDTs to generate a 3rd generation (designated version $\mathrm{O}$ ) of the E1-detecting IC RDT. The detection limit of the version-O RDT for ECSA-genotype virus was comparable to that of the version-A RDT, both against live virus $\left(1.0 \times 10^{\wedge} 4 \mathrm{pfu} / \mathrm{mL}\right)$ and against pseudotyped vectors $(6 \mathrm{ng} / \mathrm{mL}$ of HIV-1 capsid protein) (Figs. 3 and 4). The improved sensitivity of the version-B RDT for Asian- and WA-genotype viruses was retained in the version-O RDT (Figs. 3 and 4).

As described previously, Asian-genotype CHIKV carries an aspartic acid (D) at position 350 of the 6 K-E1 protein, whereas certain ECSA-genotype CHIKVs carry a glutamic acid (E) at the corresponding position; this substitution is responsible for the decreased sensitivity of RDT version A for Asiangenotype CHIKVs [28]. The sensitivities of the 3rd generation of the E1 detection RDT (version O) to each genotype were not affected by this amino acid substitution (Fig. 4).

Furthermore, we did not detect any cross-reactivity of the version-O RDT with SINV at a titer $10^{\wedge} 7 / \mathrm{mL}(7.8$ mAbs), while the version-A RDT showed slight crossreactivity at the same titer of SINV (43.9 mAbs).

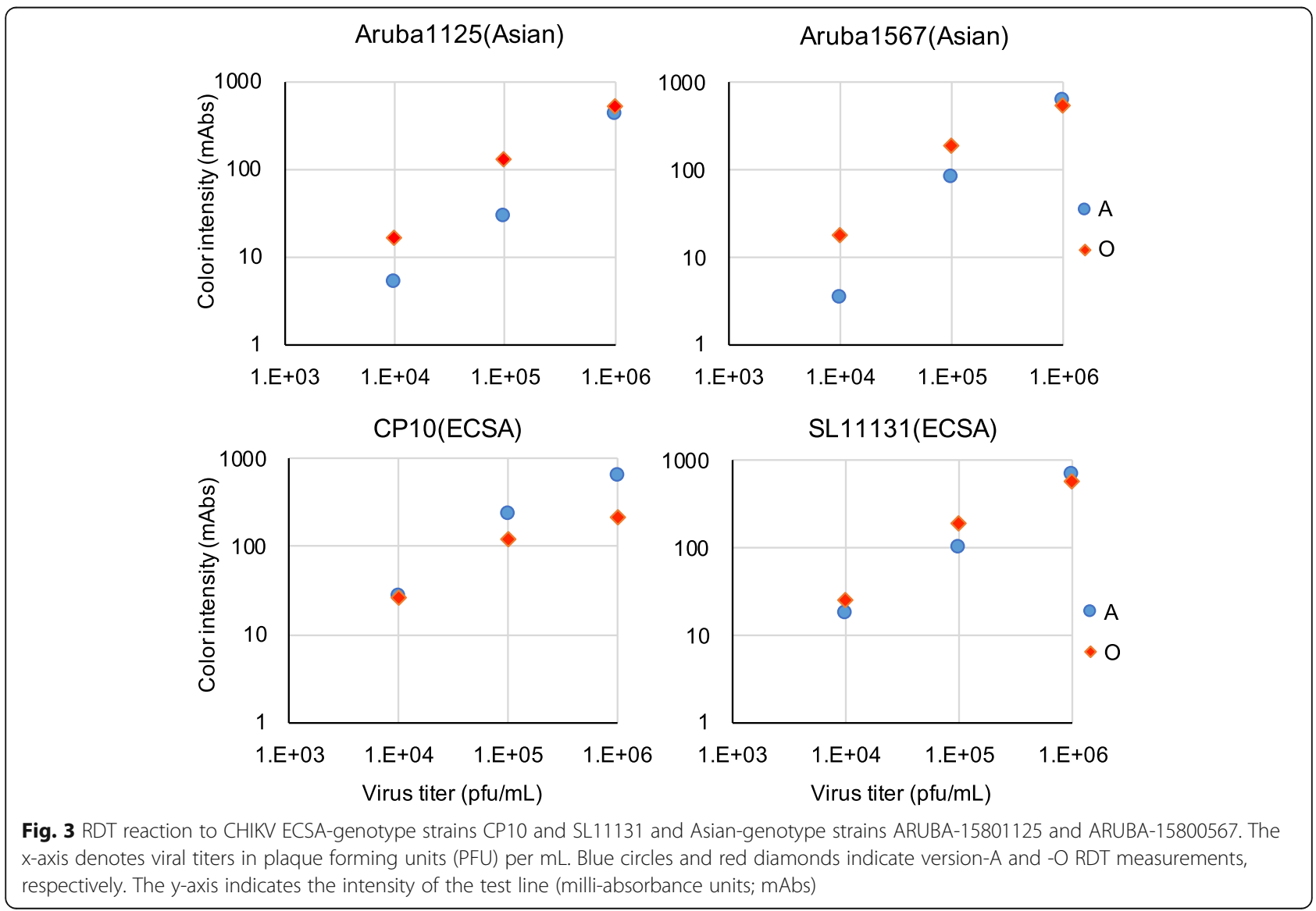


WA (350D)

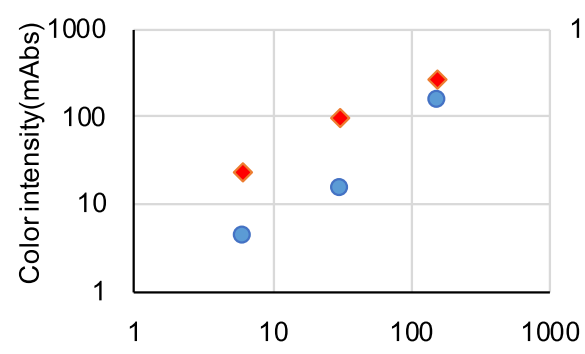

Asian (350D)

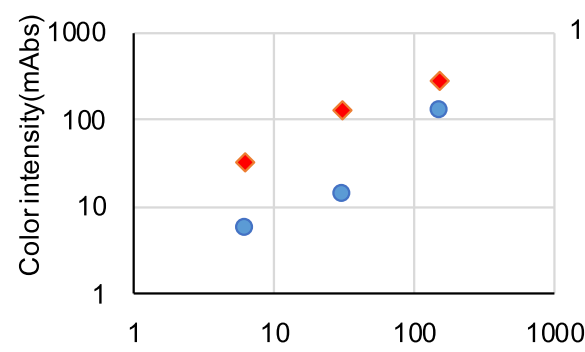

ECSA (350E)

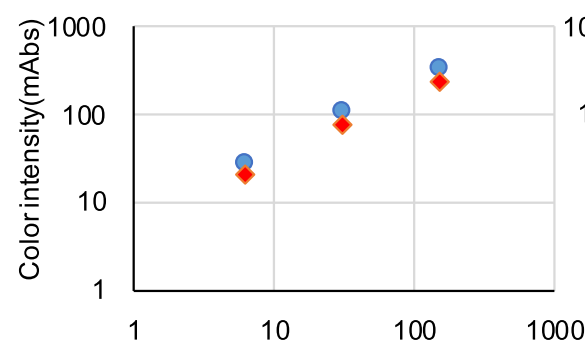

WA (350E)

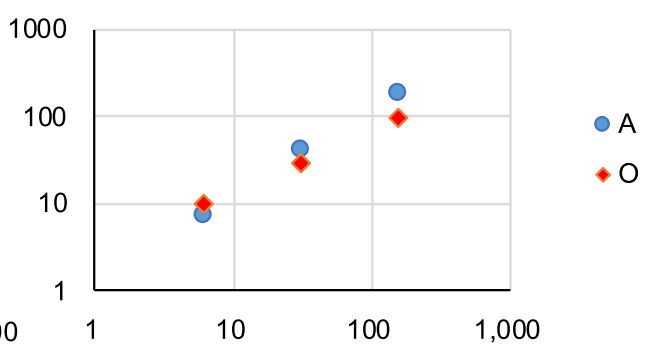

Asian (350E)

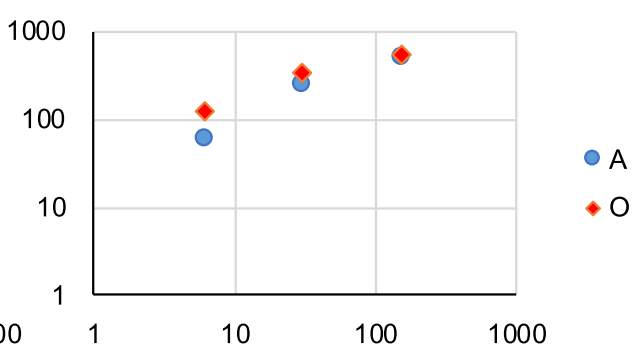

ECSA (350D)

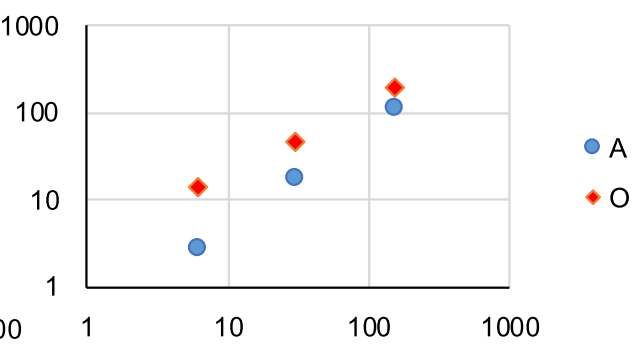

\section{p24 (ng/ml)}

Fig. 4 RDT reaction to pseudotyped lentiviruses bearing CHIKV envelope proteins with substitutions at residue 350. Pseudotyped lentiviruses bearing CHIKV envelope proteins from ECSA-genotype strain CP10, Asian-genotype strain CK12-686, WA-genotype strain 37,997, or mutant envelope proteins encoding glutamic acid-to-aspartic acid (CP10) or aspartic acid-to-glutamic acid (CK12-686 and 37,997) substitutions at residue 350 of the 6 K-E1 polyprotein were prepared at three concentrations of HIV-1 capsid protein (150, 30, and $6 \mathrm{ng} / \mathrm{mL}$ ). Blue circles and red diamonds indicate version-A and -O RDT measurements, respectively. The $y$-axis indicates the intensity of the test line (milli-absorbance units; mAbs)

\section{Trial of 3rd-generation RDT version O with CHIKV patient}

\section{sera in Bangladesh}

Since the volumes of sera from Aruba island, especially those of CHIKV PCR-positive ones, were limited, we decided to perform testing using clinical samples from other CHIKV-endemic areas. We accessed serum samples in Apollo Hospitals Dhaka, Bangladesh, where molecular detection of CHIKV and DENV was performed by RT-PCR screening during the 2017 CHIKV epidemic in Dhaka. These DENV positive sample sets were used for evaluating the specificity of the CHIKV detection RDT. As shown in Table 3, the version-O RDT exhibited 92\% (92 out of 100) sensitivity and 100\% (100 out of
Table 3 Evaluation of 3rd-generation CHIKV E1 detection RDT version $O$ in clinical samples

\begin{tabular}{cccccc}
\hline PCR & \multicolumn{1}{l}{ IC RDT } & & Sensitivity & Specificity & OAA \\
\cline { 2 - 5 } & $(+)$ & $(-)$ & & & \\
\hline$(+)^{\mathrm{a}}$ & 92 & 8 & 92 & 100 & \\
$(-)^{\mathrm{b}}$ & 0 & 100 & & \\
\hline
\end{tabular}

Sensitivity: percentage of matches between results of PCR and IC RDT in PCR positive samples

Specificity: percentage of matches between results of PCR and IC RDT in PCR negative samples

$O A A$ overall agreement

a 99 anti-CHIKV IgM-negative samples and one anti-CHIKV IgM-positive sample bdengue PCR-positive samples 
100) specificity in this setting. Among 210 CHIKV RTPCR-negative and DENV RT-PCR-negative samples identified in Dhaka, 104 were anti-CHIKV IgM positive. We further investigated 93 of these 104 anti-CHIKV IgM-positive cases and found that three (3\%) yielded positive results using our E1-antigen IC RDT (Additional file 7).

\section{Discussion}

In the present study, using newly obtained MAbs [29], we developed new IC CHIKV-E1 antigen detection RDTs with improved sensitivity to Asian- and WAgenotype viruses compared to the previous version-A RDT [28]. Although we tested only 5 samples of Asiangenotype CHIKV-infected cases (i.e., Aruban cases before anti-CHIKV IgM/IgG seroconversion), the 2nd generation CHIKV antigen detection RDT version $B$ yielded positive results in 4 of these 5 samples (i.e., $80 \%$ sensitivity). Sample numbers are low in this Asian-CHIKV serum panel, but the results suggest increased sensitivity over the previous version of RDT [27]. Furthermore, the 3rd generation RDT version O showed sensitivity of $92 \%$ (92 out of 100, 95\%-CI 85.0-95.9) in sera from 100 ECSA-genotype CHIKV-infected patients from Bangladesh. The version-O RDT also showed higher sensitivity to cultured Asian- and WA genotype CHIKVs than the version-A RDT did. These results suggest that the version-O RDT will be better for worldwide application compared to the version-A and -B RDTs.

As described in Materials and Methods, the sampling dates after the onset of fever were median 7 days and the median $\mathrm{Ct}$ value and its IQR of CHIKV-detecting PCR were 35.29 (34.22-35.50) for the Aruba samples. The sampling days after the onset of fever did not differ significantly among 11 PCR-positive and RDT-positive (median 6 days), 15 PCR-positive and RDT-negative (median 7 days), and 9 PCR-negative and RDT-positive groups (median 7 days). Importantly, many of the samples from Aruba were collected after anti-CHIKV antibody seroconversion had occurred. We look forward to confirming our findings in a larger sample size of Asiangenotype CHIKV-infected patients, preferably before seroconversion. After seroconversion, detection of antiCHIKV antibodies can be used for diagnosis. Therefore, it would be ideal to simultaneously detect both CHIKV antigen and anti-CHIKV antibodies for accurate diagnosis.

In terms of the false-positive rate in CHIKV PCRnegative samples, the specificity of the version-O RDT in samples in Bangladesh was $100 \%$, while that of the version-B RDT was $83.3 \%$ (45 out of 54) in samples from Aruba. Specificity of the version-B RDT in the Aruba samples was $94.2 \%$ (33 out of 35) for anti-CHIKV IgMand IgG-negative cases, while that in anti-CHIKV IgM- or IgG-positive cases was 63.2\% (12 out of 19) (Table 2). As described above, the version-B RDT uses 3 clones of anti-CHIKV MAbs, while the version-O RDT uses 5 MAbs, including 3 MAbs used in the version-B RDT. Thus, it is unlikely that a specific component present only in the version-B RDT caused the false-positive reactions. Most of the Ct-values of CHIKV-detecting RTPCR in Bangladesh samples were lower than 20, and the median Ct-value and its IQR was 17.02 (14.89-19.92) (see Additional file 7). Of course, it is not appropriate to directly compare Ct-values between Aruba and Dhaka patients given that these values were determined with different real-time PCR systems, but these $\mathrm{Ct}$-values suggest that the CHIKV sera in Dhaka were collected earlier in the disease course. Results of ELISA detecting antiCHIKV IgM also support this idea, since only one out of 100 CHIKV PCR-positive cases was anti-CHIKV IgM positive (Table 3, Additional file 7). In contrast, among 54 PCR-negative samples from Aruba, 19 were positive for either anti-CHIKV IgM or IgG (Table 2, Additional file 3). As the kinetics of chikungunya antigenemia are not well understood, it is possible that the IC RDTpositive but PCR-negative samples are not truly CHIKV negative. In other words, CHIKV structure proteins might have persisted longer than the viral genome in blood, since the structural proteins might be expressed from a sub-genomic or defective viral RNA without fulllength genomic RNA replication. In fact, persistence of a defective alphavirus genome in infected cells has been reported previously [37]. Indeed, three (3\%) positive results were obtained when we tested 93 CHIKV PCRnegative and DENV PCR-negative but anti-CHIKV IgMpositive samples (Additional file 7).

The precise mechanisms associated with chronic CHIKV-associated joint disease are largely unknown, although the effects of abnormalities present prior to infection cannot be ruled out. In CHIKV-infected patients, high viral loads are common, and anti-CHIKV IgM or IgG has been observed to persist in patients for 18 months or longer $[38,39]$. These findings suggest that continuous immune stimulation, possibly by persistent or continuously expressed CHIKV antigens, could play a role in prolonged $\mathrm{CHIKV}$-associated poly-arthralgia, although no tools are available to rapidly detect CHIKV antigens. It will be interesting to use the diagnostic RDT described in the present work for follow-up studies on antigen persistence in post-chikungunya chronic polyarthralgia. Thus, it will be critical to further evaluate these false-positive cases using our revised CHIKV RDT.

\section{Conclusion}

We developed a new CHIKV E1-antigen rapid diagnostic test with improved sensitivity (compared to the previous version) to the Asian and WA genotypes of CHIKV. 
Although the revised RDT needs to be validated against larger panels of sera with varying CHIKV genotypes, the new RDT holds a promise for use in endemic areas for ECSA-genotype CHIKV encoding a $6 \mathrm{~K}$-E1 protein with an E350 residue, viruses that are currently spreading from South Asia to South East Asia [36, 40].

\section{Supplementary information}

Supplementary information accompanies this paper at https://doi.org/10. 1186/s12985-020-01364-4

Additional file 1. Selection of monoclonal antibodies (MAbs) for CHIKV detection rapid diagnostic test. (A) Means and standard deviations of signal/background ratios for selection of MAbs against E1 protein. AuNPs column indicates MAb conjugated with AuNPs and membrane column indicates MAb adsorbed onto nitrocellulose membrane. Green: Signal/ background ratio was lower than 1.0 (strong non-specific reaction or no signal). Orange: Signal/background ratio was between 1.0 and 2.0 (faint). Red: Signal/background ratio was higher than 2.0 (strong positive). Yellow: Signal/background ratio was higher than 2.0 but standard deviation was high. (B) Means of signal/background ratios for selection of MAbs against capsid protein. AuNPs column indicates MAb conjugated with AuNPs and membrane column indicates MAb adsorbed onto nitrocellulose membrane. Green: Signal/background ratio was lower than 1.0 (strong non-specific reaction or no signal). Orange: Signal/background ratio was between 1.0 and 2.0 (faint). Red: Signal/background ratio was higher than 2.0 (strong positive)

Additional file 2. RDT reaction to CHIKV Asian-genotype and ECSAgenotype strains. ECSA-genotype strain CP10 and Asian-genotype strains ARUBA-15801567 (ARUBA1567) and ARUBA-15801125 (ARUBA1125) were grown in Vero cells. The $x$-axis denotes viral titer in plaque forming units (PFU) /mL. Blue circles, orange squares, and gray diamonds indicate CP10, ARUBA1125, and ARUBA1567 measurements, respectively. The $y$-axis indicates the intensity of the test line (milli-absorbance units; mAbs).

Additional file 3. Data of Aruba patients. ND: not determined. Additional file 4. Evaluation of CHIKV E1 detection RDT version B in anti-CHIKV IgM or lgG-positive clinical samples. CHIKV E1 detection RDT version B were evaluated in 34 anti-CHIKV IgM-positive and 31 lgGpositive clinical samples. OAA: overall agreement.

Additional file 5. Comparison of CHIKV E1 detection RDT versions A and $B$ in 20 clinical samples. OAA: overall agreement.

Additional file 6. Evaluation of CHIKV E1 detection RDT version B in 60 clinical samples. OAA: overall agreement.

Additional file 7. Data of Dhaka patients. ND: not determined.

E1(mAbs): mili absorbance units of CHIKV E1 antigen

immunochromatogaraphic rapid diagnostic test (version O). Positive results in CHIKV E1 detection, anti-CHIKV IgM, dengue virus NS1, antidengue virus $\lg M$ and $\lg G$ are highlighted with red.

\section{Abbreviations}

CHIKV: Chikungunya virus; ECSA: East/Central/ South African; WA: West African; IC: Immunochromatorgraphic; RDT: Rapid diagnostic test; MAb: Monoclonal antibody; SINV: Sindbis virus; DENV: Dengue virus; IOL: Indian Ocean lineage; mAbs: Milli-absorbance units; RT-PCR: Reverse transcription-polymerase chain reaction; OAA: Overall agreement

\section{Acknowledgements}

We are grateful to all the patients who participated in this study. We also thank Dr. Jan Jacobs for his critical discussion on this manuscript and Dr. Tahmina Sultana, Ms. Sasicha Somboon and Ms. Kumi Yamamoto for their assistance.

\section{Authors' contributions}

KS: creation of the test RDT devices, acquisition of data, draft the manuscript. $\mathrm{RH}$ : design of the work, acquisition of data, interpretation of data, substantively revised the manuscript. JP: acquisition of data. AT: acquisition of data. EEN: acquisition of data, interpretation of data, draft and substantively revised the manuscript. RB: acquisition of data, sampling. BB: acquisition of data, substantively revised the manuscript. LC: acquisition of data, substantively revised the manuscript. RR: acquisition of data. $\mathrm{AH}$ : acquisition of data. HI: supply the test RDT devices. PL: design of the work, interpretation of data. ME: supervision, interpretation of data, substantively revised the manuscript. MR: design of the work, interpretation of data. TS: conception, design of the work, substantively revised the manuscript. The author(s) read and approved the final manuscript.

\section{Funding}

This work was supported by Japan Agency for Medical Research and Development (AMED) JP19fm0108003 to TS. The funders had no role in study design, data collection and analysis, decision to publish, or preparation of the manuscript.

\section{Availability of data and materials}

All data generated or analyzed during this study are included in this published article and its supplementary information files.

\section{Ethics approval and consent to participate}

The study proposal was approved by the Research and Ethical Practice Committee of Institute of Tropical Medicine, Antwerp (Approval No. 1013/15) and Apollo Hospitals Dhaka (Approval No. ERC 16/2018-1). This study was exempt from obtaining participants' consent since only leftover specimens were used after anonymization.

\section{Consent for publication}

Not applicable.

\section{Competing interests}

$\mathrm{KS}$ and $\mathrm{HI}$ are paid employees from TANAKA Kikinzoku Kogyo. TANAKA Kikinzoku Kogyo created the immunochromatographic devices and supplied them to Institute of Tropical Medicine Antwerp and Apollo Hospitals Dhaka for evaluation. TANAKA Kikinzoku Kogyo had no role in study design, data collection and analysis, decision to publish, or preparation of the manuscript.

\section{Author details}

${ }^{1}$ Research Institute for Microbial Diseases, Osaka University, Suita, Japan. ${ }^{2}$ POCT Products Business Unit, TANAKA Kikinzoku Kogyo K.K, Hiratsuka, Japan. ${ }^{3}$ Department of Clinical Sciences, Institute of Tropical Medicine, Antwerp, Belgium. ${ }^{4}$ Mahidol-Osaka Center for Infectious Diseases, Mahidol University, Bangkok, Thailand. ${ }^{5}$ Department of Microbiology and Immunology, Mahidol University, Bangkok, Thailand. 'andslaboratorium Aruba, Oranjestad, Aruba. ${ }^{7}$ Apollo Hospitals Dhaka, Dhaka, Bangladesh.

Received: 24 December 2019 Accepted: 23 June 2020

Published online: 02 July 2020

\section{References}

1. Ross RW. The Newala epidemic. III. The virus: isolation, pathogenic properties and relationship to the epidemic. J Hyg (Lond). 1956;54:177-91.

2. Lumsden $\mathrm{WH}$. An epidemic of virus disease in Southern Province, Tanganyika territory, in 1952-53. II. General description and epidemiology. Trans R Soc Trop Med Hyg. 1955;49:33-57.

3. Zeller H, Van Bortel W, Sudre B. Chikungunya: its history in Africa and Asia and its spread to new regions in 2013-2014. J Infect Dis. 2016;214:S436-s40.

4. Powers AM, Brault AC, Tesh RB, Weaver SC. Re-emergence of Chikungunya and O'nyong-nyong viruses: evidence for distinct geographical lineages and distant evolutionary relationships. J Gen Virol. 2000;81:471-9.

5. Rougeron V, Sam IC, Caron M, Nkoghe D, Leroy E, Roques P. Chikungunya, a paradigm of neglected tropical disease that emerged to be a new health global risk. J Clin Virol. 2015;64:144-52.

6. Schuffenecker I, Iteman I, Michault A, Murri S, Frangeul L, Vaney MC, Lavenir R, Pardigon N, Reynes JM, Pettinelli F, et al. Genome microevolution of chikungunya viruses causing the Indian Ocean outbreak. PLoS Med. 2006;3: e263.

7. Arankalle VA, Shrivastava S, Cherian S, Gunjikar RS, Walimbe AM, Jadhav SM, Sudeep AB, Mishra AC. Genetic divergence of Chikungunya viruses in India (1963-2006) with special reference to the 2005-2006 explosive epidemic. J Gen Virol. 2007;88:1967-76. 
8. Mavalankar D, Shastri P, Raman P. Chikungunya epidemic in India: a major public-health disaster. Lancet Infect Dis. 2007;7:306-7.

9. Rianthavorn P, Prianantathavorn K, Wuttirattanakowit N, Theamboonlers A, Poovorawan Y. An outbreak of chikungunya in southern Thailand from 2008 to 2009 caused by African strains with A226V mutation. Int J Infect Dis. 2010;14(Suppl 3):e161-5.

10. Duong V, Andries AC, Ngan C, Sok T, Richner B, Asgari-Jirhandeh N, Bjorge S, Huy R, Ly S, Laurent D, et al. Reemergence of Chikungunya virus in Cambodia. Emerg Infect Dis. 2012;18:2066-9.

11. Sam IC, Loong SK, Michael JC, Chua CL, Wan Sulaiman WY, Vythilingam I, Chan SY, Chiam CW, Yeong YS, AbuBakar S, Chan YF. Genotypic and phenotypic characterization of Chikungunya virus of different genotypes from Malaysia. PLoS One. 2012;7:e50476.

12. Rezza G, Nicoletti L, Angelini R, Romi R, Finarelli AC, Panning M, Cordioli P, Fortuna C, Boros S, Magurano F, et al. Infection with chikungunya virus in Italy: an outbreak in a temperate region. Lancet. 2007;370:1840-6.

13. Grandadam M, Caro V, Plumet S, Thiberge JM, Souares Y, Failloux AB, Tolou HJ, Budelot M, Cosserat D, Leparc-Goffart I, Despres P. Chikungunya virus, southeastern France. Emerg Infect Dis. 2011;17:910-3.

14. Nunes MR, Faria NR, de Vasconcelos JM, Golding N, Kraemer MU, de Oliveira LF, Azevedo Rdo S, da Silva DE, da Silva EV, da Silva SP, et al. Emergence and potential for spread of Chikungunya virus in Brazil. BMC Med. 2015;13: 102.

15. Charlys da Costa A, Theze J, SCV K, Sanz-Duro RL, MRL F, LCC M, IMO B, LEC S, MAL N, Moura AA, et al. Spread of Chikungunya Virus East/Central/South African Genotype in Northeast Brazil. Emerg Infect Dis. 2017;23:1742-4.

16. Leparc-Goffart I, Nougairede A, Cassadou S, Prat C, de Lamballerie X. Chikungunya in the Americas. Lancet. 2014;383:514.

17. Sahadeo NSD, Allicock OM, De Salazar PM, Auguste AJ, Widen S, Olowokure B, Gutierrez C, Valadere AM, Polson-Edwards K, Weaver SC, Carrington CVF. Understanding the evolution and spread of chikungunya virus in the Americas using complete genome sequences. Virus Evol. 2017:3:vex010.

18. Lanciotti RS, Valadere AM. Transcontinental movement of Asian genotype chikungunya virus. Emerg Infect Dis. 2014;20:1400-2.

19. Khan K, Bogoch I, Brownstein JS, Miniota J, Nicolucci A, Hu W, Nsoesie EO, Cetron M, Creatore Ml, German M, Wilder-Smith A. Assessing the origin of and potential for international spread of chikungunya virus from the Caribbean. PLoS Curr. 2014:6.

20. Nsoesie EO, Kraemer MU, Golding N, Pigott DM, Brady OJ, Moyes CL, Johansson MA, Gething PW, Velayudhan R, Khan K, et al. Global distribution and environmental suitability for chikungunya virus, 1952 to 2015. Euro Surveill. 2016;21.

21. Thiberville SD, Moyen N, Dupuis-Maguiraga L, Nougairede A, Gould EA, Roques $P$, de Lamballerie $X$. Chikungunya fever: epidemiology, clinical syndrome, pathogenesis and therapy. Antivir Res. 2013;99:345-70.

22. Schwartz O, Albert ML. Biology and pathogenesis of chikungunya virus. Nat Rev Microbiol. 2010;8:491-500.

23. Morrison TE. Reemergence of chikungunya virus. J Virol. 2014;88:11644-7.

24. Waggoner JJ, Ballesteros G, Gresh L, Mohamed-Hadley A, Tellez Y, Sahoo MK, Abeynayake J, Balmaseda A, Harris E, Pinsky BA. Clinical evaluation of a single-reaction real-time RT-PCR for pan-dengue and chikungunya virus detection. J Clin Virol. 2016;78:57-61.

25. Okabayashi T, Sasaki T, Masrinoul P, Chantawat N, Yoksan S, Nitatpattana N, Chusri S, Morales Vargas RE, Grandadam M, Brey PT, et al. Detection of chikungunya virus antigen by a novel rapid immunochromatographic test. J Clin Microbiol. 2015;53:382-8.

26. Jain J, Okabayashi T, Kaur N, Nakayama E, Shioda T, Gaind R, Kurosu T, Sunil S. Evaluation of an immunochromatography rapid diagnosis kit for detection of chikungunya virus antigen in India, a dengue-endemic country. Virol J. 2018;15:84.

27. Huits R, Okabayashi T, Cnops L, Barbe B, Van Den Berg R, Bartholomeeusen K, Arien KK, Jacobs J, Bottieau E, Nakayama EE, et al. Diagnostic accuracy of a rapid $\mathrm{E} 1$-antigen test for chikungunya virus infection in a reference setting. Clin Microbiol Infect. 2018;24:78-81.

28. Tuekprakhon A, Nakayama EE, Bartholomeeusen K, Puiprom O, Sasaki T, Huits R, Luplertlop N, Kosoltanapiwat N, Maneekan P, Arien KK, et al. Variation at position 350 in the Chikungunya virus 6K-E1 protein determines the sensitivity of detection in a rapid E1-antigen test. Sci Rep. 2018;8:1094.

29. Tuekprakhon A, Puiprom O, Sasaki T, Michiels J, Bartholomeeusen K, Nakayama EE, Meno MK, Phadungsombat J, Huits R, Arien KK, et al. Broadspectrum monoclonal antibodies against chikungunya virus structural proteins: promising candidates for antibody-based rapid diagnostic test development. PLoS One. 2018;13:e0208851.

30. Sasayama M, Benjathummarak S, Kawashita N, Rukmanee P, Sangmukdanun S, Masrinoul P, Pitaksajjakul P, Puiprom O, Wuthisen P, Kurosu T, et al. Chikungunya virus was isolated in Thailand, 2010. Virus Genes. 2014;49:485-9.

31. Puiprom O, Morales Vargas RE, Potiwat R, Chaichana P, Ikuta K, Ramasoota $P$, Okabayashi T. Characterization of chikungunya virus infection of a human keratinocyte cell line: role of mosquito salivary gland protein in suppressing the host immune response. Infect Genet Evol. 2013;17:210-5.

32. Huits R, De Kort J, Van Den Berg R, Chong L, Tsoumanis A, Eggermont K, Bartholomeeusen K, Arien KK, Jacobs J, Van Esbroeck M, et al. Chikungunya virus infection in Aruba: diagnosis, clinical features and predictors of postchikungunya chronic polyarthralgia. PLoS One. 2018;13:e0196630.

33. Phadungsombat J, Tuekprakhon A, Cnops L, Michiels J, Van Den Berg R, Nakayama EE, Shioda T, Arien KK, Huits R. Two distinct lineages of chikungunya virus cocirculated in Aruba during the 2014-2015 epidemic. Infect Genet Evol. 2019;104129.

34. Baer A, Kehn-Hall K. Viral Concentration Determination Through Plaque Assays: Using Traditional and Novel Overlay Systems. Jove J Vis Exp. 2014: e52065.

35. Kishishita N, Takeda N, Anuegoonpipat A, Anantapreecha S. Development of a Pseudotyped-Lentiviral-vector-based neutralization assay for Chikungunya virus infection. J Clin Microbiol. 2013;51:1389-95.

36. Rahman M, Yamagishi J, Rahim R, Hasan A, Sobhan A. East/central/south African genotype in a Chikungunya outbreak, Dhaka, Bangladesh, 2017. Emerg Infect Dis. 2019;25:370-2.

37. Weiss $B$, Levis R, Schlesinger $S$. Evolution of virus and defective-interfering RNAs in BHK cells persistently infected with Sindbis virus. J Virol. 1983;48: $676-84$.

38. Grivard P, Le Roux K, Laurent P, Fianu A, Perrau J, Gigan J, Hoarau G, Grondin N, Staikowsky F, Favier F, Michault A. Molecular and serological diagnosis of Chikungunya virus infection. Pathol Biol (Paris). 2007:55:490-4.

39. Malvy D, Ezzedine K, Mamani-Matsuda M, Autran B, Tolou H, Receveur MC, Pistone T, Rambert J, Moynet D, Mossalayi D. Destructive arthritis in a patient with chikungunya virus infection with persistent specific $\lg M$ antibodies. BMC Infect Dis. 2009;9:200

40. Intayot P, Phumee A, Boonserm R, Sor-Suwan S, Buathong R, Wacharapluesadee S, Brownell N, Poovorawan Y, Siriyasatien P. Genetic Characterization of Chikungunya Virus in Field-Caught Aedes aegypti Mosquitoes Collected during the Recent Outbreaks in 2019, Thailand. Pathogens. 2019:8.

\section{Publisher's Note}

Springer Nature remains neutral with regard to jurisdictional claims in published maps and institutional affiliations.

Ready to submit your research? Choose BMC and benefit from:

- fast, convenient online submission

- thorough peer review by experienced researchers in your field

- rapid publication on acceptance

- support for research data, including large and complex data types

- gold Open Access which fosters wider collaboration and increased citations

- maximum visibility for your research: over $100 \mathrm{M}$ website views per year

At BMC, research is always in progress.

Learn more biomedcentral.com/submissions 